\title{
Densidad, clase social y apoyo expresivo*
}

\author{
Irene Cruz ${ }^{1}$, Joan Miquel Verd- QUIT ${ }^{ \pm}$- Universitat Autònoma de Barcelona
}

\section{Resumen}

En el análisis de redes personales, la oposición entre redes bonding y bridging ha estado en el centro de varias de las hipótesis que relacionan la estructura de las redes con el apoyo disponible en ellas. La densidad ha sido uno de los indicadores más recurrentes para medir las redes bonding o close-knit dada su facilidad de operacionalización e interpretación. Con todo, el ajuste de la densidad como medida del carácter bonding o bridging de las redes no ha sido contrastado con una muestra amplia y aleatoria de redes personales con un número de contactos mayor al de las sub-redes de apoyo o de confianza. Usando una muestra de 433 redes personales de 30 Alteri cada una, este artículo explora la correlación entre la densidad, el apoyo expresivo intercambiado y la posición de clase de los Ego en el contexto de la estructura social catalana. Los resultados sugieren que la densidad sería mejor comprendida como una variable dependiente de la estabilidad y las transiciones vitales reflejadas en las redes personales puesto que su relación con el apoyo inscrito en las redes es débil.

Palabras clave: Densidad - Apoyo expresivo - Clase social - Fuerza del vínculo.

\begin{abstract}
In personal network analysis, the opposition between bonding and bridging networks has been at the core of most hypotheses relating network structure and support embedded in it. Due to its ease of operationalization and interpretation, density has been one of the most common indicators used for measuring bonding or close-knit networks. Nevertheless, the reliability of density as a measure of bonding networks has never been contrasted against a large and random sample of personal networks containing contacts beyond the support sub-network. Using a large sample of ego-centred networks $(n=433)$ of 30 alteri each, this paper explores the correlation between density, expressive support exchanged, and Ego's class position within the Catalan social structure. Results suggest that density should preferably be used as a dependent variable related to stability and life transitions reflected in personal networks since it's relationship with support embedded in a personal network is weak.
\end{abstract}

Key words: Density - Expressive support - Social class - Strength of tie.

\footnotetext{
${ }^{1}$ Con el apoyo del Comissionat per a Universitats i Recerca del DIUE de la Generalitat de Catalunya i del Fons Social Europeu.

* Este artículo forma parte del proyecto CSO2008-01470 del Ministerio de Educación y Ciencia. Los autores de este artículo forman parte de dicho proyecto. Agradecemos los comentarios recibidos por los participantes en el seminario "Inmigración, Redes y Cohesión Social" celebrado los días 16 y 17 de Diciembre en la Universidad Autónoma de Barcelona, especialmente las sugerencias de Carlos Lozares, Michel Grossetti, y Ainhoa de Federico.

${ }^{ \pm}$Centre d'Estudis Sociològics sobre la Vida Quotidiana i el Treball (QUIT). Departament de Sociologia irene.cruz@uab.cat, joanmiquel.verd@uab.cat.
} 


\section{Introducción}

De acuerdo con John Scott, la densidad es una de las medidas estructurales más usadas y al mismo tiempo peor utilizadas en sociología (2000). Como indicador, informa de la proporción de relaciones existentes sobre el total de relaciones posibles. Es una medida global que resume en un solo porcentaje cuán cerca o lejos se encuentra la red del estado completo o estado en que todos los nodos tuvieran conexión entre ellos. Su sencillez, informatividad y versatilidad permiten que la medida sea empleada en muchas temáticas distintas. Por ejemplo, de los 24 artículos publicados en la revista Redes en el 2010, 15 hacen algún uso o referencia a la densidad. Sin embargo, el indicador parece haber adquirido una serie de connotaciones teóricas que lo asocian a la idea de relaciones próximas y fuertes sin que acabe de estar justificado ni teórica ni empíricamente.

El objetivo de este artículo consiste en revisar dos hipótesis recurrentes en la literatura que emplea la densidad en el análisis de redes personales: las que asocian el indicador a características socio-demográficas de los Ego, como la clase social, y las que lo asocian a características de las relaciones entre Ego y sus Alteri, como la disposición al apoyo expresivo. Parece que ambas derivas conceptuales proceden de la tesis sobre la fuerza de los lazos débiles (en adelante SWT por sus siglas en inglés) planteada por Mark Granovetter en 1973 y las sucesivas revisiones que se le han hecho (entre otros Lin, Ense y Vaughn, 1981; Marsden y Campbell, 1984; Blau, 1991; Montgomery, 1992). Pese a tener más de 40 años, la teoría goza de buena salud, a juzgar por la cantidad de referencias que se le hacen todavía. Por ejemplo, el índice de publicaciones académicas Sociological Abstracts arroja 907 citas a la tesis SWT, las 50 primeras de las cuales son publicaciones del año 2010, y 51 de las cuales lo usan como palabra clave. Estrictamente, Granovetter no usa la medida de la densidad para caracterizar a los lazos fuertes y débiles, sin embargo se vale frecuentemente del indicador para construir su argumento sobre el papel de los lazos débiles en las redes personales; en sus propias palabras: "This is close to saying, in my terms, that one's strong ties form a dense network, one's weak ties a less dense one." (1973, p. 1370).

En las siguientes páginas plantearemos primero por qué resulta necesario revisar los supuestos conceptuales y los usos que se derivan del indicador de la densidad, expondremos los datos usados para los análisis, ofreceremos los principales resultados del contraste, y a modo de cierre, recogeremos las principales conclusiones y reflexiones. 


\section{La densidad: usos conceptuales}

En primer lugar, revisaremos las hipótesis que asocian la densidad de las redes personales con la clase social y otros atributos socio-demográficos con efectos de estructuración social (como el nivel de estudios o la continuidad/discontinuidad laboral en los últimos dos años). La idea central de esta hipótesis, que no es como Granovetter originalmente plantea el efecto de los lazos débiles en la circulación de la información, podría resumirse en que las personas con más recursos profesionales, económicos o sociales tienen mayor capacidad para mantener contactos dispersos y poco transitivos en sus redes personales; y de modo inverso, las personas en situaciones sociales más desfavorecidas tienen mayor dependencia social y material de sus contactos más próximos, por lo que se ven envueltas en redes mucho más densas. Por ejemplo, varios de los autores citados por Granovetter en su revisión de la teoría de los lazos débiles (1983) abordan la relación entre pobreza y lazos fuertes en redes densas. Entre ellos Ericksen y Yancey (1977) plantean que las redes "fuertes" están asociadas a la inseguridad económica y a la falta de servicios sociales y que los individuos con niveles de estudios menores son más propensos a usar contactos cercanos para buscar empleo. Stack (1974) y Lomnitz (1977) realizaron sendos estudios etnográficos sobre poblaciones muy diferentes, el primero en un gueto urbano de población afroamericana y el segundo en un barrio de chabolas en la periferia de México DF, llegando a conclusiones similares sobre el papel central de los lazos fuertes en la vida y la supervivencia de los más desfavorecidos.

Anteriormente, Homans (1950, también citado por Granovetter) planteó que son los individuos de mayor estatus dentro de un grupo los que tienen mayor facilidad de mantener contactos fuera del mismo, por lo tanto, de tener más relaciones no transitivas. Ya Elizabeth Bott en 1957 asocia la densidad de la red (o en sus términos, connectedness) a la clase social. En su hipótesis las clases medias y altas, al tener mayor movilidad geográfica por razones laborales, tendrían relaciones sociales menos densas que las familias de clase obrera, más arraigadas a un contexto local y familiar. La hipótesis de Bott abre toda una línea de investigación sobre la relación entre la densidad de las relaciones familiares y de las amistades con éstas, la posición de clase y los roles de esposas y maridos en los matrimonios, que dará pie a diversas variaciones sobre el uso del indicador de la densidad y su relación con la clase social (ver por ejemplo Piselli, 2009; Ishii-Kuntz y Maryanski, 2003, 1992; Dasgupta, 1992; Bocker, 1988; Hill, 1985, 1988; Ericksen, Yancey y Ericksen, 1979; Chatterjee, 1976). 
Lecturas más recientes de esta hipótesis se plantean en términos considerablemente similares a los usados por Granovetter en 1983. Por ejemplo, Wenhong Zhang (2004) realizó un estudio sobre el efecto de la estructura de clases sobre las redes personales de población residente en Beijing. Una de sus conclusiones principales es que las clases profesionales y administrativas tienen redes más grandes y con más lazos débiles que la población de clase obrera. Zhang atribuye las diferencias observadas a "las distintas posiciones de clase que las personas tienen en la estructura social y económica", en tanto que los trabajadores en posiciones sociales más altas "tienen más oportunidades para conocer y asociarse con personas dentro y fuera de su propia clase" (p. iv), de acuerdo a un modelo de oportunidades y restricciones. Otras variaciones recientes argumentan que los jóvenes (particularmente los de estudios universitarios) tienen menor densidad en sus redes por el hecho de mantener relaciones sociales en distintos entornos (Degenne y Lebeaux, 2005) o porque la prolongación del tiempo de estudio permite prolongar también durante mayor tiempo formas de sociabilidad juvenil (Bidart y Lavenu, 2005).

Planteamos la necesidad de revisar los supuestos sobre la relación entre la densidad y la posición en la estructura social puesto que provienen principalmente de trabajos realizados en los años setenta. Desde entonces, las trayectorias laborales de una parte importante de los trabajadores y trabajadoras han perdido en estabilidad y linealidad (Alonso, 2000). Trayectorias laborales inestables o marcadas por la temporalidad posiblemente muestren niveles de densidad bajos, al acumular contactos no necesariamente transitivos. Por otra parte, la hipótesis que revisamos fue formulada en clave masculina, bajo un modelo "male bread winner" de relación estable y lineal con el mercado laboral. Especialmente entre los estudios menos recientes en los que se ha puesto a prueba la tesis SWT, se trabaja con muestras exclusivamente masculinas (por ejemplo Granovetter, 1973; Lin, Ensel y Vaughn, 1981; una de las muestras de Marsden y Campbell, 1984). También por esta razón parece oportuno revisar los supuestos que relacionan la densidad a la posición de clase.

Por otra parte, argumentaremos que el uso de la densidad (baja) como medida de los lazos débiles es fruto de una deriva conceptual que debe someterse a revisión. No es lo mismo un lazo informativamente débil que estructuralmente aislado. 
El segundo conjunto de hipótesis que revisaremos son las que asocian la densidad con dimensiones más expresivas de las relaciones como el apoyo personal (véanse ejemplos de este uso en Haines, Hurlbert y Beggs, 2008, 1996; McPherson, Popielartz y Drobnic, 1992) o la cohesión social entendida como confianza mutua (como ejemplo en la tradición de Putnam, 1995, también en Portes, 1998; y en Falci, 2009 como ejemplo aplicado). La asociación de ideas suele basarse en la oposición clásica entre redes close knit y loose-knit (Bott, 1957), o, en términos más actuales, redes bonding (Woolcock 1998, Woolcock y Narayan, 1999) y bridging (Granovetter, 1973). Las redes bonding (entendidas como redes de relaciones densas, homófilas, duraderas e intensas, en las que la mayoría de las personas se conocen unas a otras) se asocian con las relaciones de apoyo interpersonal y emocional, la multiplexidad y la confianza. Por otra parte, las redes bridging (en la que pocos de los contactos de Ego se conocen entre sí, formando componentes separados y en las que, se supone, hay mayor presencia de lazos débiles) suelen asociarse con la circulación de informaciones nuevas y el acceso a contactos nuevos (Granovetter, 1973; McLanahan, 1981). La densidad en esta ocasión se usa como variable independiente, como predictor de la fuerza de los lazos.

Detectamos que en la literatura se establecen estas asociaciones entre densidad y apoyo porque ambos fenómenos tienden a ocurrir simultáneamente: las sub-redes de confianza y de apoyo suelen ser muy densas. Del mismo modo, a la idea de cohesión también se suele yuxtaponer la noción de confianza. Pero la cohesión entendida como densidad de relaciones al nivel estructural debe distinguirse conceptualmente de la cohesión entendida como las cualidades del vínculo que une a los nodos, ya sean la confianza o el apoyo mutuo. Es inherente a la tesis sobre la fuerza de los lazos débiles de Granovetter (1973) la idea de que las relaciones más cercanas de ego, sus lazos fuertes, son más densas entre sí, tienen mayor nivel de solapamiento, por el principio de la transitividad de los lazos fuertes. También argumenta el autor que es con las relaciones más cercanas con quienes se intercambia en mayor medida apoyo de tipo expresivo, de lo cual se derivaría la conclusión lógica que es con los contactos más densos con quienes se intercambia en mayor medida el apoyo expresivo. La conclusión está justificada y se ha contrastado empíricamente en distintas ocasiones (ver por ejemplo Stoloff, Glanville y Bienenstock, 1999, y McLanahan, Wedemeyer y Adelberg, 1981, que analizan el papel clave del apoyo de familiares y amistades en las tareas de cuidado de menores para la participación de las mujeres con hijos e hijas en el mercado 
laboral; Rogero-García, 2009, relaciona la proximidad afectiva de mayores dependientes con sus cuidadores y cuidadoras con el uso de servicios formales de asistencia). Sin embargo, nuestra hipótesis es que lo que resulta claro al nivel de díada y tríada no tiene por qué ser extensible al conjunto de la red; es decir, las redes con una densidad global más alta no presentarían necesariamente mayores ocasiones de intercambio de apoyo expresivo, si se trata de redes que se extiendan más allá de la sub-red de confianza y apoyo. La densidad puede ser fruto de diversas situaciones, que no necesariamente conllevan la dimensión expresiva.

Finalmente, buscaremos dar respuesta a la pregunta de si la densidad es o no una buena medida del carácter bonding de las redes personales.

\section{Datos}

Los datos con los que trabajamos proceden del proyecto I+D+I Estudio comparado de casos sobre la influencia mutua entre capital e integración sociales y la inserción, estabilidad, promoción y cualificación en el empleo (CASREDIN) con referencia CSO2008-01470, subvencionado por el Ministerio de Educación y Ciencia dentro del marco del VI Plan Nacional de Investigación Científica, Desarrollo e Innovación Tecnológica 2008-2011, el investigador principal del cual es Carlos Lozares. El artículo firmado por el conjunto del equipo en este mismo volumen (Lozares, López, Verd et al., 2011) expone los detalles del muestreo y la metodología seguidas en el trabajo de campo. Sintéticamente, el muestreo ha sido aleatorio y estratificado. La estratificación bietápica se ha hecho primero por localidades y luego por colectivos de población que responden a un análisis factorial realizado sobre el censo de Cataluña del 2001 (ver López Roldán y Lozares, 2008). Los datos consisten en 433 encuestas de redes personales con 4 bloques de preguntas: un primer bloque de preguntas de caracterización socio-demográfica de los Ego, la pregunta generadora de redes de 30 Alteri, un tercer bloque de preguntas de caracterización socio-demográfica de cada Alter mencionado y por último las relaciones existentes entre cada par de Alteri. Las redes personales se construyen a partir de relaciones de conocimiento mutuo (se puede ver la pregunta generadora y el conjunto del cuestionario en el Anexo de Lozares et al., 2011).

De acuerdo con Christopher McCarthy (2002) 30 Alteri es un tamaño de red personal que permite recoger tanto el conjunto de contactos más próximos como otro tipo de contactos menos cercanos, optimizando los costes de la recogida de información. Por ello, la muestra con la que trabajamos parece particularmente 
apropiada.

El método empleado para contrastar las hipótesis ha consistido en análisis estadísticos bivariados y multivariados, elegidos en función del tipo de variables empleadas. El método se corresponde con la segunda modalidad de análisis de redes personales propuesta en Lozares et al. (2011).

\section{Variables}

Las variables empleadas hacen referencia a información de los Ego, a no ser que se indique lo contrario. La variable de la densidad es el indicador estructural que mide la proporción de lazos existentes sobre el total de lazos posibles. La medida de clase social que se ha empleado corresponde a una recodificación en tres categorías (Alta, Media, Baja) de la variable de Categoría Socio-profesional. Ésta se construyó originalmente como una adaptación (de 11 categorías) de la variable empleada en la Encuesta de Población Activa desde 1994 (de 19 categorías). La variable de Nivel de estudios se ha recodificado en cuatro categorías: Sin estudios, Obligatorios, Medios, Superiores. La variable sobre la Dis/continuidad laboral en los últimos dos años se ha construido a partir de las variables sobre situación laboral actual y situación laboral hace dos años, con las siguientes categorías originales: estudiante que no trabaja, estudiante que trabaja, jubilado/a/ pensionista/ incapacitado/a, gestión del hogar/tareas de cuidado, en paro, trabajo a tiempo completo y con contrato indefinido, trabajo a tiempo parcial y/o con contrato no indefinido, otras situaciones. La variable resultante sobre dis/continuidad tiene 4 categorías: continuidad activa, discontinuidad en la actividad, incorporación a la actividad y continuidad inactiva. La variable sobre el apoyo expresivo es un recuento del total de Alteri con los que Ego afirma intercambiar apoyo en problemas personales, ponderada según el cómputo total de ocasiones con las que Ego intercambia cualquier tipo de ayuda con su red, con tal de corregir posibles sesgos cognitivos en las respuestas. Porcentaje de amistades en la red, porcentaje de familia directa y porcentaje de familia extensa son variables que designan la proporción de relaciones de cada tipo que Ego nombra en su red, como variables continuas. La variable Tipo de relación: familiares informa sobre la relación que cada Alter mantiene con Ego, poniendo especial atención en las relaciones familiares. Las variables sobre proximidad afectiva provienen de una escala de valoración de la proximidad de la relación entre Ego y Alter, que va del 0 (nada próximo) al 5 (Somos íntimos). Hemos usado la escala como variable relativa a cada uno de los Alteri y también hemos usado las categorías como porcentajes de relaciones más o menos próximas en cada red. Hemos usado una variable de 
Estado Civil en un sentido amplio de pareja, que no se restringe a las relaciones formales de matrimonio. Por último, se han empleado las variables de sexo y edad. Esta última se ha usado como variable continua y por intervalos de 30 años.

\section{Resultados}

\subsection{Relación entre densidad y medidas de clase social}

En la tabla 1 se muestran los resultados de los análisis de varianza (ANOVA) efectuados sobre el grupo de variables relacionadas con la clase social. Densidad se ha tomado como variable dependiente. En conjunto, observamos que los estadísticos $\mathrm{F}$ tienen valores bajos que se explican por la alta variabilidad de la densidad dentro las categorías de las variables empleadas y por el tamaño de la muestra. Si bien tanto las diferencias por clase social como por nivel de estudios son significativas al 95\%, el test Scheffé de comparaciones múltiples permite ver que las diferencias de medias entre los grupos o categorías de las variables no son significativas. Esto queda reflejado en el número de subconjuntos homogéneos formados a partir del análisis: para cada una de las dos variables señaladas se distingue un único subconjunto.

Las diferencias de densidad por niveles de estudios que hemos observado no se explican por una proporción distinta de relaciones de amistad en la red (para ningún grupo de edad). 


\begin{tabular}{|c|c|c|c|}
\hline Variable & $\mathbf{F}$ & Densidad Media & $\begin{array}{l}\text { no subconjuntos } \\
\text { homogéneos }\end{array}$ \\
\hline $\begin{array}{l}\text { Categoría Socio- } \\
\text { Profesional }\end{array}$ & $3.906 * *$ & & \\
\hline Alta & & 38.0123 & 1 \\
\hline Media & & 43.0940 & 1 \\
\hline Baja & & 37.9678 & 1 \\
\hline Nivel de estudios & $4.734 * *$ & & \\
\hline Sin estudios & & 46.0533 & 1 \\
\hline Obligatorios & & 44.9716 & 1 \\
\hline Medios & & 38.8561 & 1 \\
\hline Superiores & & 38.1101 & 1 \\
\hline $\begin{array}{c}\text { Trayectoria laboral - } 2 \\
\text { años }\end{array}$ & $12.612 * * *$ & & \\
\hline continuidad activa & & 38.3930 & 1,2 \\
\hline $\begin{array}{l}\text { discontinuidad en la } \\
\text { actividad }\end{array}$ & & 35.5152 & 1 \\
\hline $\begin{array}{l}\text { incorporación a la } \\
\text { actividad }\end{array}$ & & 48.8741 & 2,3 \\
\hline continuidad inactiva & & 50.1865 & 3 \\
\hline \multicolumn{4}{|c|}{$\begin{array}{l}* * * \text { significativo al } 99 \% \\
* * \text { significativo al } 95 \% \\
\text { Fuente: elaboración propia según dados de CASREDIN }\end{array}$} \\
\hline
\end{tabular}

Tabla 1. Resultados ANOVAs entre medidas de posición social y densidad

La variable de Dis/continuidad laboral en los dos últimos años da resultados significativos al $99 \%$ y $\mathrm{F}$, sin ser alta, es considerablemente mayor que en el resto de variables. También es la única variable en la que se forman subconjuntos diferenciados por la variabilidad entre las categorías. Éstas tienen diferencias entre sí en cuanto a edad media, proporción de hombres y mujeres y nivel de estudios, aunque no tienen una identificación clara con perfiles sociales determinados, guardando cierta diversidad interna. A partir del test de Scheffé distinguimos dos grandes grupos: los que vienen de o mantienen una trayectoria de actividad y los que vienen de o mantienen una trayectoria inactiva. 
El fenómeno del desempleo no puede entenderse como una realidad neutra en términos de género, sino que es una más de las ausencias femeninas en el mercado laboral, resultado de la desigual división sexual del trabajo (Torns, 2001). Por ello hemos revisado que estas diferencias de densidad por dis/continuidades laborales no escondieran en realidad un sesgo de género en la relación con el mercado laboral. Sin embargo, nuestros datos dan a entender que las densidades de hombres y mujeres varían similarmente entre las distintas categorías de la variable sobre dis/continuidad laboral, como puede observarse en el Gráfico 1.

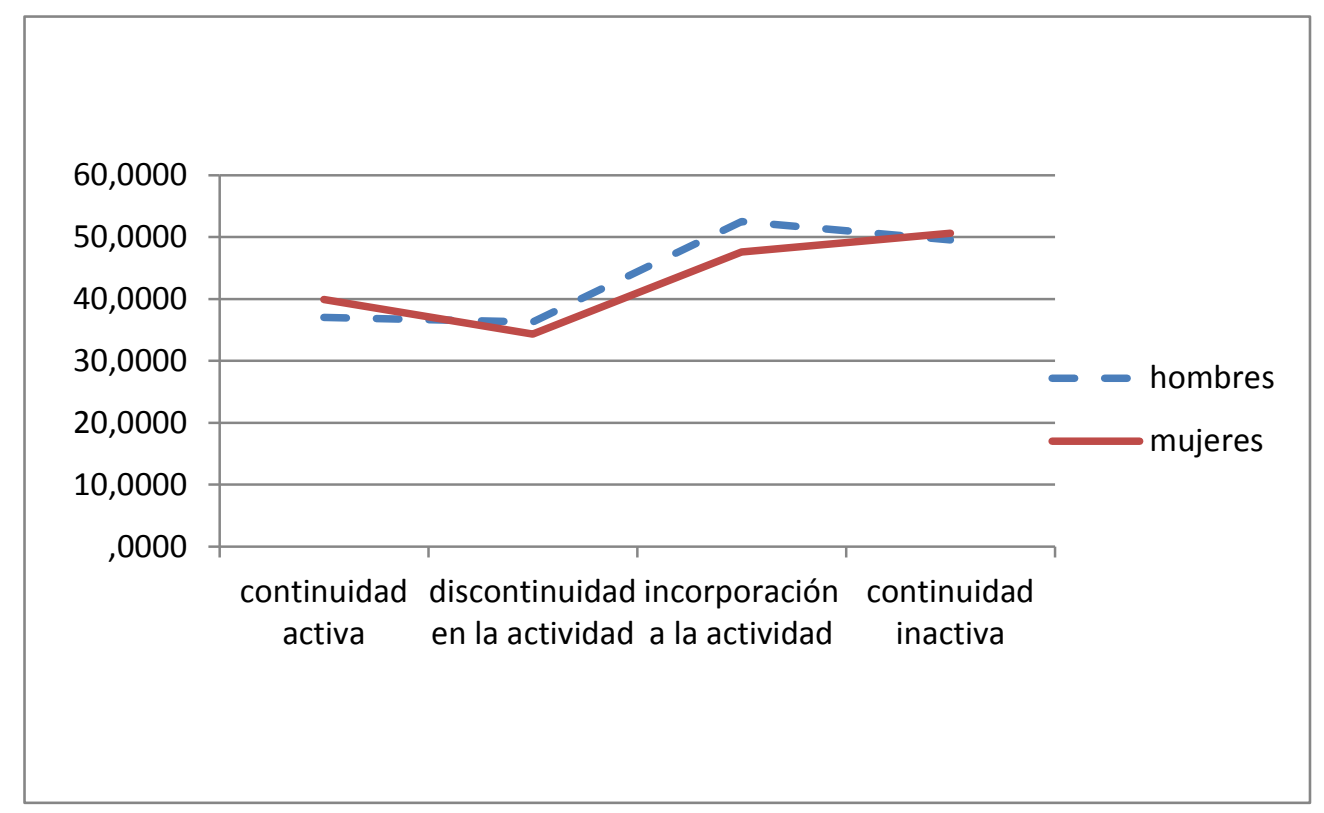

Gráfico 1. Variaciones en la densidad global por dis/continuidad laboral y sexo.

Con los análisis precedentes, venimos viendo que el indicador de la densidad en las redes no se comporta como cabría esperar de un indicador de lazos débiles. Marsden y Campbell (1984) en su artículo Measuring tie strength, concluyen que la mejor medida de la fuerza de un lazo es precisamente la valoración de Ego sobre la fuerza del lazo. Estaríamos de acuerdo con esta conclusión si por fuerza del vínculo se entiende la dimensión expresiva de la relación, es decir, la propia valoración de Ego sobre la fuerza del lazo, ni más ni menos.

Para nuestros datos, la correlación de orden cero entre la densidad y el porcentaje de relaciones en la red valoradas como "no próxima", es de $-0.261 * * *$, es decir, a mayor densidad en las redes, menor proporción de relaciones con quienes no se siente proximidad afectiva. Esta correlación es un dato relevante y significativo, pero la asociación es demasiado baja como para tomar la densidad como medida de los contactos débiles en este sentido expresivo del término. 
Por otra parte, cabe preguntar qué podría aclarar la densidad mejor que el estatus social. Si la densidad se asocia a las relaciones próximas y como tal, transitivas, cabría esperar que el tipo de relación que une a Ego con sus Alteri proporcione mayor información acerca de esta característica estructural. La correlación entre densidad y porcentaje de Alteri que son familia de Ego es de $0.495^{* * *}$ y con el porcentaje de familia extensa es de $0.450 * * *$. Es decir, que el rol adscrito a la relación entre Ego y a Alter (hermanos, amigas, vecinos, compañeras de trabajo...) parece ser más informativo sobre la estructura de la red que meramente los atributos de Ego.

La presencia de relaciones familiares en la red está influenciada por la edad de Ego y por su estado civil (ver Tabla 2). La correlación de orden cero entre la edad y la densidad es de $0.191 * * *$, que es una asociación baja aunque significativa. Sin embargo, en un análisis más detallado se observa que esta asociación es espuria y está mediada por el estado civil: al usar como filtro el estado civil en la correlación entre la densidad y la edad, $\mathrm{Chi}^{2}$ deja de ser significativo tanto entre los solteros como entre los casados, y sus asociaciones bajan hasta 0,028 en los dos grupos. Por lo tanto entendemos que la asociación entre densidad y estado civil, así como la propia relación espuria entre densidad y edad, denotan que el cambio de estado civil supone una forma de transición en la vida que deja una huella en la red en forma de los contactos que se mantienen y los que se alejan. La densidad se comprendería entonces como una variable dependiente de estas transiciones.

\begin{tabular}{|c|c|c|c|}
\hline Edad & Hasta $\mathbf{3 0}$ & Ente $\mathbf{3 1}$ y $\mathbf{6 0}$ & $\mathbf{6 1} \mathbf{~}+$ \\
\hline Estado Civil & 33 & 40 & 47 \\
\hline & $\begin{array}{c}\text { Sin pareja, } \\
\text { divorciado/a. }\end{array}$ & En pareja, casado/a & Viudo/a \\
\hline & 30 & 43 & 53 \\
\hline
\end{tabular}

Tabla 2. Porcentaje de relaciones familiares según edad y estado civil

\subsection{Relación entre densidad y apoyo expresivo.}

La correlación entre la densidad de la red (usada como variable independiente) y la medida ponderada de la frecuencia con que Ego intercambia relaciones de apoyo expresivo con sus Alteri es de 0.216*** y queda ilustrada en el gráfico 2 . Se intuye cierta concentración alrededor de la diagonal principal, pero la relación entre las variables no puede considerarse más que de tipo débil: una regresión simple muestra que $R^{2}=0.044$, es decir, la densidad de la red sólo da cuenta de un $4,4 \%$ de la variabilidad del apoyo expresivo. A nuestro entender, esto plantea un 
interrogante a la comprensión y el uso de la densidad como medida, o siquiera aproximación, a la dimensión expresiva de las redes personales.

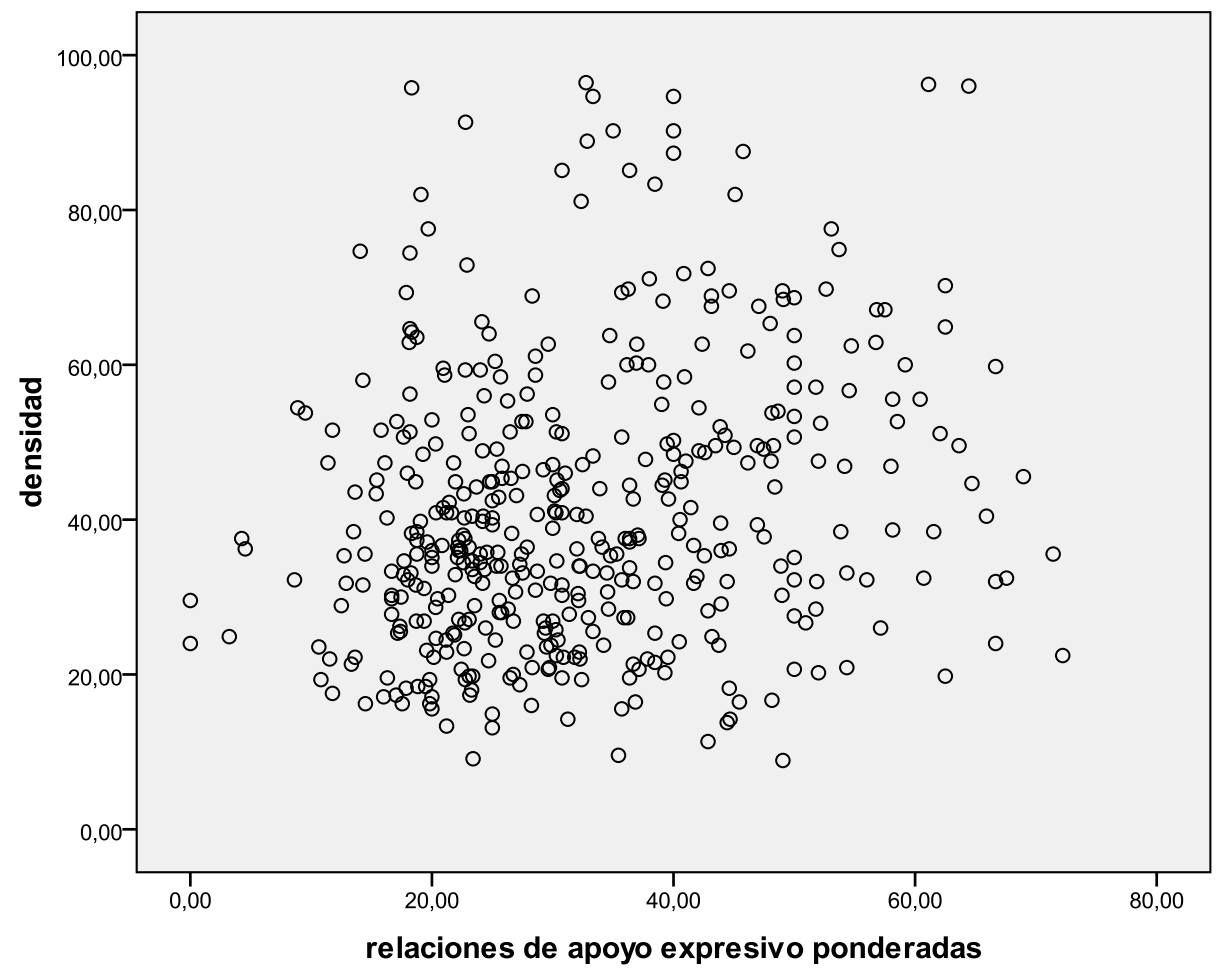

Gráfico 2. Dispersión del apoyo expresivo según densidad global.

La correlación entre una medida de intensidad de los vínculos (porcentaje de Alteri a quienes se considera íntimos) y la densidad de la red es de $0.236 * * *$. Una regresión lineal simple arroja una $\mathrm{R}^{2}=0.053$, es decir, que la cantidad de vínculos íntimos en la red sólo explicaría el 5,3\% de la densidad. Si bien la asociación es significativa y relevante, valoramos que es muy insuficiente para tomar la densidad como medida de la proximidad afectiva y viceversa, o en otras palabras, podríamos decir que la densidad no parece la medida más adecuada de la dimensión bonding o del carácter expresivo que a menudo se le atribuye.

Agneessens, Waege y Lievens (2006) sugieren que las relaciones de apoyo se explican mejor por el rol del contacto o el marco normativo que rige el tipo de relación entre la díada. Por ejemplo, en caso de enfermedad, es más probable recurrir al cónyuge o a las hijas adultas que a las amistades; en cambio, es más probable recurrir a las amistades para hablar de preocupaciones o al buscar compañía. Esta hipótesis es consistente con los resultados que hemos obtenido en la sección anterior. 


\section{Conclusiones}

En las páginas precedentes se han revisado algunas hipótesis implícitas en dos usos frecuentes del indicador de la densidad en la literatura del análisis de redes personales.

En relación al vínculo entre densidad y clase social, se ha visto que la asociación entre las variables es débil, con lo que no se ha podido confirmar la hipótesis según la cual las personas de clases altas o medias tengan redes con una estructura menos densa que las personas de clases trabajadoras. Aparece con más claridad el efecto de la estabilidad en el empleo con la estructura de las relaciones, lo que confirma la importancia del entorno laboral como ámbito de sociabilidad. Para las personas activas, el entorno laboral suele constituir la principal fuente de relaciones fuera de la familia. Hemos visto también que la inactividad tiene efectos similares sobre las redes de hombres y de mujeres, aumentando la proporción de contactos familiares y de la densidad global de la red. Los resultados del análisis de los efectos del estado civil nos han llevado a plantear la hipótesis de que las diferencias en la densidad de las redes informan mejor sobre procesos de cambio, momentos de transición en la vida de las personas, que de atributos más estables o lineales como la clase social y la edad. Esto lleva a plantear una primera línea para continuar la investigación, que es ver la huella de los momentos de transición como el nacimiento de un hijo, el fin de los estudios, el paso al paro, casarse o la jubilación en la red de contactos, mediante la longevidad de las relaciones y los componentes que forman, en la línea del trabajo de Bidart y Lavenu (2005), Degenne y Lebeaux (2005).

Trabajar con el concepto de densidad resulta problemático porque lo que originalmente era un indicador sobre la proporción de contactos existentes ha adquirido una serie de connotaciones teóricas que arrastran cierta confusión. Hemos visto que la densidad, como uno de los indicadores recurrentes para caracterizar las redes de lazos fuertes o "close-knit", se asocia débilmente con los contenidos expresivos de las redes como la disposición al apoyo mutuo o la proximidad afectiva. Esto nos lleva a cuestionar el uso de la densidad como medida o aproximación de la fuerza de los lazos. Planteamos que los resultados que hemos obtenido son diferentes a los que se puedan haber observado previamente porque hemos trabajado con redes más amplias que las sub-redes de confianza, y que al incluir más tipos de relaciones, aquello que es cierto a nivel de díada (las personas con quienes se tiene relaciones más cercanas y más transitivas es con quienes se 
intercambia más apoyo) no tiene por qué serlo a nivel global. Sin embargo, sí se ha detectado que la densidad se asocia al tipo de vínculo que une a la díada (proporción de relaciones familiares), en línea con lo propuesto por Agneessens (2006).

Finalmente, extraemos algunas reflexiones a raíz de observar que la densidad no se comporta del modo previsto para una medida de la proximidad/distancia de los vínculos en un sentido expresivo del término ni tampoco responde a la idea de apoyo mutuo que cabría suponer de las relaciones más próximas. Valoramos que es preferible usar medidas atributivas para caracterizar las dimensiones expresivas de la cercanía/distancia o fuerza/debilidad de los lazos y las redes. Con el trabajo expuesto no nos podemos pronunciar acerca del interés de la densidad como medida estrictamente estructural del carácter Bonding o Bridging de las redes. Para tal fin, sería deseable ampliar el ejercicio de Marsden y Campbell (1984) en Measuring Tie Strength y comparar sistemáticamente distintas baterías de indicadores estructurales y atributivos que se hayan empleado en la literatura especializada para caracterizar las relaciones fuertes y débiles. Lo que hemos concluido con nuestros datos es que el indicador global de la densidad no es una buena medida de la dimensión expresiva, ya sea fuerte o débil, de las relaciones.

\section{Bibliografía}

Agneessens, Filip; Waege, Hans; Lievens, John. (2006). "Diversity in social support by role relations: A typology". Social Networks 28 (2006) 427-441. Consulta [0608-10]

Alonso, Luís Enrique. (2000). Trabajo y posmodernidad: el empleo débil. Madrid: Fundamentos.

Bidart, Claire; Lavenu, Daniel. (2005): "Evolution of personal networks and life events", Social Networks, 27: 359-376. Consulta [25-03-10]

Blau, Judith. (1991). "When weak ties are structured". En Blau \& Goodman (eds.). (1991). Social roles and social institutions : essays in honor of Rose Laub Coser. Boulder: Westview press.

Bocker, Anita. (1988). "A Reexamination of Bott's Hypothesis with Regard to Turkish Migrants". Sociologische Gids, vol. 35, no. 5, pp. 320-331, Sept-Oct. Consulta [12-08-10]

Bott, Elizabeth. (1957). Family and social network: roles, norms, and external 
relationships in ordinary urban families. London : Tavistock, 1968

Chatterjee, Mary. (1976). "Conjugal Roles and Social Networks in an Indian Urban Sweeper Locality". Journal of Marriage and the Family, vol. 39, no. 1, pp. 193-202, Feb 1976. Consulta [11-08-10]

Dasgupta, Sathi. (1992). "Conjugal Roles and Social Network in Indian Immigrant Families: Bott Revisited". Journal of Comparative Family Studies, vol. 23, no. 3, pp. 465-480, autumn 1992. Consulta [11-08-10]

Degenne, Alain; Lebeaux, Marie-Odile (2005): "The dynamics of personal networks at the time of entry into adult life", Social Networks, 27: 337-358. Consulta [25-0310]

Ericksen, Julia A., Yancey, William L., Ericksen, Eugene P. (1979). "The Division of Family Roles". Journal of Marriage and Family, Vol. 41, No. 2 (May, 1979), pp. 301313. Consulta [03-07-10]

Falci, Christina; McNeely, Clea. (2009). "Too Many Friends: Social integration, Network Cohesion and Adolescent Depressive Symptoms". Social Forces 87(4):2031-62, June 2009. Consulta [11-11-10]

Granovetter, Mark (1973). "The strength of weak ties". The American Journal of Sociology. Vol. 78, no 6 (Mayo), pp. 1360-1380. Consulta [20-09-10]

Granovetter, Mark. (1983). "The strength of weak ties: a network theory revisited". Sociological Theory, Vol. 1, pp. 201-233. Consulta [20-09-10]

Haines, Valerie A., Hurlbert, Jeanne S., Beggs, John J. (1996). "Exploring the Determinants of Support Provision: Provider Characteristics, PersonalNetworks, Community Contexts, and Support Following Life Events" Journal of Health and Social Behavior, Vol. 37, No. 3 (Sep., 1996), pp. 252-264. Consulta [01-09-10]

Haines, Valerie A., Hurlbert, Jeanne S., Beggs, John J. (2008). "Contextualizing Health outcomes: do effects of network structure differ for women and men?". Sex Roles, no 59:164-175. Consulta [20-11-10]

Hill, Malcolm D. (1988). "Class, Kinship Density, and Conjugal Role Segregation". Journal of Marriage and the Family, vol. 50, no. 3, pp. 731-741, Aug 1988. Consulta [25-11-10]

Hill, Malcolm D.(1985). "The Effects of Social Norms and Kinship Interaction on Conjugal Role Organization". Sociological Viewpoints, vol. 1, no. 1, pp. 1-7, 1985. 
Consulta [25-11-10]

Ishii-Kuntz, Masako; Maryanski, A. R.(2003). "Conjugal Roles and Social Networks in Japanese Families". Journal of Family Issues, vol. 24, no. 3, pp. 352-380, Apr 2003. Consulta [11-08-10]

Lin, Nan; Ensel, Walter; Vaughn, John. (1981). "Social resources and strength of ties: structural factors in occupational status attainment". American Sociological Review, Vol. 46, no 4, pp. 393-405. [20-09-10]

Lopez Roldán Pedro; Lozares Carlos. (2008). "La construcción de la muestra. El trabajo de campo de la Encuesta de condiciones de vida y hábitos de la población de Catalunya" Metodologies i Recerques. IERMB, Barcelona, no 1:17-39.

Lozares, Carlos; Verd, Joan Miquel; López-Roldán, Pedro; Martí, Joel y José Luis Molina (2011). "Cohesión, Vinculación e Integración sociales como formas de Capital social", REDES-Revista hispana para el análisis de redes sociales, vol. 20 \#1 (http://revista-redes.rediris.es), enviado.

Marsden, Peter; Campbell, Karen. (1984). "Measuring tie strength". Social forces, vol. 63, n02, pp. 482-501. Consulta [08-10-10]

Maryanski, Alexandra; Ishii-Kuntz, Masako. (1992). "Conjugal Roles and Social Networks in Japanese Families". American Sociological Association, 1992. Consulta [11-08-10]

McCarty, Christopher. (2002). "Structure in Personal networks". Journal of Social Structure. Vol. 3, no 1. Consulta [02-06-10]

McLanahan, Sara; Wedemeyer, Nancy V.; Adelberg, Tina. (1981). "Network Structure, Social Support, and Psychological Well-Being in the Single-Parent family". Journal of Marriage and Family, Vol. 43, No. 3 (Aug., 1981), pp. 601-612. Consulta [16-05-10]

McPherson, M.; Popielarz, P.; Drobnic, S. (1992). "Social networks and organizational dynamics". American Sociological review. Vol. 57, no 2: 153-170. Consulta [23-11-09]

Montgomery, James. (1992). "Job search and network composition: implications of the strength of weak ties hypothesis". American Sociological review, vol. 57, no 5, pp. 586-596. [02-06-10]

Piselli, Fortunata. (2009)."Network Analysis in the Study of Gender Differences". International Review of Sociology/Revue Internationale de Sociologie, vol. 19, no. 
1, pp. 147-154, Mar 2009. Consulta [28-10-10]

Portes, Alejandro. (1998). "Social Capital: Its Origins and Applications in Modern Sociology". Annual Review of Sociology, Vol. 24 (1998), pp. 1-24. Consulta [2211-08]

Putnam, Robert. (1995). "Bowling Alone: America's Declining Social Capital". Journal of Democracy, January 1995, pp. 65-78. Consulta [13-05-09]

Rogero-García, Jesús. (2009). "Distribución en España del cuidado formal e informal a las personas de 65 y más años en situación de dependencia". Revista Española de Salud Pública, 83:393-405. Consulta [21-10-10]

Scott, John. (2000), Social network analysis : a handbook . London: Sage, 2a ed.

Stoloff, Jennifer; Glanville, Jennifer; Bienenstock, Elisa. (1999). "Women's participation in the labor force: the role of social networks". Social Networks, vol. 21, pp. 91-108. [11-08-10]

Woolcock, M. (1998), 'Social capital and economic development: Toward a theoretical synthesis and policy framework', Theory and Society, vol. 27, no. 2, pp. 151-208.

Woolcock, M; Narayan, D. (1999). Social capital: implications for development theory, research, and policy. [on-line]. Disponible en: http://wbro.oxfordjournals.org/content/15/2/225.short

Zhang, Wenhong. (2004). "Class Structure and Social Networks in Urban China". Dissertation Abstracts International, A: The Humanities and Social Sciences, vol. 64 , no. 11 , pp. 4229-A-4230-A, May 2004. Consulta [13-12-10] 